\title{
Model animals and their applications
}

\author{
GAO Xiang \\ MOE Key Laboratory of Model Animal for Disease Study, Model Animal Research Center, Nanjing University, Nanjing 210061, China
}

Received March 11, 2015

Citation: $\quad$ Gao X. Model animals and their applications. Sci China Life Sci, 2015, 58: 319-320, doi: 10.1007/s11427-015-4842-3

Looking back a decade ago when I decided to use "model animal" to name our newly established institute, my outrageous boldness can only be described by the old Chinese slang, "newborn calf could never sense the danger of tiger". Happily, my courageous belief, along with the hard work of my colleagues at Model Animal Research Center of Nanjing University, paid off eventually. We have witnessed the great progress in this research field in China in the past 10 years. This issue of Science China Life Sciences samplings some of these accomplishments.

The model animals are selected because they are not only suitable for addressing some important biological questions, but particularly efficient for certain manipulations. As one of the oldest "model animals", Xenopus still contributes greatly to understand the choice between pluripotency and differentiation during germ layer formation, as described by Cao Ying [1] in this issue. Another example is Drosophila that contributed greatly for uncovering fundamental controls for embryonic pattern formation by EMS mutagenesis and genetic screenings. Chen Jiong's group [2] analyzed the genetic control of collective migration, a phenomenon involving many physiological and pathological processes, by knocking down 360 conserved signaling transduction genes in ovary. C. elegans is very useful for aging studies because of its short life span around only 20 days. In this issue, Chen Di [3] summarized some exciting results on fine mapping of the molecular mechanisms on prolonged life span by dietary restriction. Finally, mice are most likely be analyzed to explain the human-like physiological phenomena. Gan ZhenJi [4] presented example of this system by studying muscle

email: gaoxiang@nju.edu.cn type switching in mice, whereas Xu Ying [5] tried to conform one particular gene's function in circadian. Moreover, Zhu MinSheng [6] reported progress in developing new strains as tools for neuroscience studies.

This issue also includes some research papers and reviews on potential applications in medicine. For zebrafish, Luo LingFei [7] has written a very nice review to explain how to take advantage of optic transparence proper of this creature for better understanding the organ regeneration. I [8] described a mutant mouse strain with disrupted homeostasis in multiple systems, indicating the connection among bone, blood chemistry, and glucose metabolism. Using gene-targeting mice, Li ChaoJun [9] presented some convincing data linking human diseases with protein prenylation. Finally, we included a short clinic report by Jiang Qing [10], finding positive association between $\mathrm{ABO}$ blood type/ABO gene and susceptibility to deep vein thrombosis following orthopedic surgery. In my personal opinion, human has "evolved" to model animal category, due to the "big data" analysis on both massive genome sequencing data and comprehensive clinic diagnosis data with modern health care system.

We should never forget the importance of resource collection for model animals. In addition to the existing well-known international resource centers for flies, zebrafish, and mice, I am happy to see two papers in this issue describing the great progress in China Zebrafish Resource Center (CZRC) in Wuhan [11] and National Resource Center for Mutant Mice (NRCMM) in Nanjing [12]. While CZRC has emerged as one of the three largest centers for zebrafish strains collection in the world, NRCMM has become the major player in the newly formed International 
Mouse Phenotype Consortium (IMPC). Just remember this ambitious goal of IMPC: to generate the first comprehensive encyclopedia on mammalian gene function.

Let me conclude my introduction by mentioning the recent innovation on genome editing. The CRISPR/Cas9 technology will completely change the landscape of model animal research field. We are facing the explosion of new strains, new mutants, new phenotyping data in the near future. So it is time now to pulse our reading and start to think, what do we really want to learn from studying these fascinating lives?

1 Cao Y. Germ layer formation during Xenopus embryogenesis: the balance between pluripotency and differentiation. Sci China Life Sci, 2015, 58: 336-342

2 Luo J, Zuo JT, Wu J, Wan P, Kang D, Xiang C, Zhu H, Chen J. In vivo RNAi screen identifies candidate signaling genes required for collective cell migration in Drosophila ovary. Sci China Life Sci, 2015, 58: 379-389

3 Lan JF, Zhang X, Chen D. Molecular mechanisms of dietary restriction in aging-insights from Caenorhabditis elegans research. Sci China Life Sci, 2015, 58: 352-358

4 Liu J, Liang XJ, Gan ZJ. Transcriptional regulatory circuits control- ling muscle fiber type switching. Sci China Life Sci, 2015, 58: 321-327

5 Qu ZP, Wang XH, Liu DC, Gao X, Xu Y. Inactivation of Cipc alters the expression of Perl but not circadian rhythms in mice. Sci China Life Sci, 2015, 58: 368-372

6 Tao T, Chen C, Sun Jie, Peng YJ, Zhu MS. A bacterial artificial chromosome transgenic mouse model for visualization of neurite growth. Sci China Life Sci, 2015, 58: 373-378

7 Shi WC, Fang ZB, Li L, Luo LF. Using zebrafish as the model organism to understand organ regeneration. Sci China Life Sci, 2015, 58: $343-351$

8 Zou JH, Xiong XW, Lai BB, Sun M, Tu X, Gao X. Glucose metabolic abnormality is associated with defective mineral homeostasis in skeletal disorder mouse model. Sci China Life Sci, 2015, 58: 359-367

9 Xu N, Shen N, Wang XX, Jiang S, Xue B, Li CJ. Protein prenylation and human diseases: a balance of protein farnesylation and geranylgeranylation. Sci China Life Sci, 2015, 58: 328-335

10 Sun Y, Mao P, Lu JW, Dai J, Teng HJ, Jiang Q. ABO blood type and ABO gene with susceptibility to deep vein thrombosis following orthopedic surgery: a case-control study in Chinese Han population. Sci China Life Sci, 2015, 58: 390-391

11 Xie XW, Pan LY, Sun YH. Growing with the world: rapid development of the zebrafish research in China and the China Zebrafish Resource Center. Sci China Life Sci, 2015, 58: 396-399

12 Qi X, Gao X. Towards a better understanding of mouse and human diseases-International Mouse Phenotyping Consortium. Sci China Life Sci, 2015, 58: 392-395

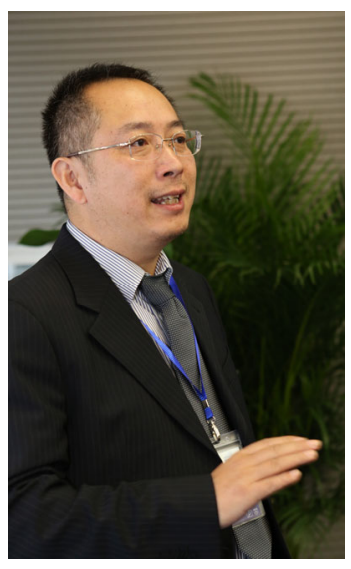

\section{Biographical Sketch}

Gao Xiang got his Ph.D. degree of anatomy and developmental biology from Thomas Jefferson University. He was then trained as postdoctoral associate at Roche Institute of Molecular Biology and The Jackson Laboratory. In 2000, He was recruited back as Professor by Nanjing University. He founded both National Resource Center of Mutant Mice in China and Model Animal Research Center of Nanjing University. His laboratory has been working on broad topics of pathogenesis using mouse models generated genome manipulation. The current focus is the molecular mechanisms for controlling physiological homeostasis, especially the metabolic homeostasis. He is the current president of Asian Mouse Mutagenesis and Resource Association. He is a Steering Committee member for International Mouse Phenotype Consortium.

Open Access This article is distributed under the terms of the Creative Commons Attribution License which permits any use, distribution, and reproduction in any medium, provided the original author(s) and source are credited. 